Clinics Review articles: Cardiology Clinics: Nephrocardiology

Ed P. Hatamizadeh

Aug 2021; p307

\title{
Uremic toxins and cardiovascular system
}

Sophie Valkenburg, MSc

Griet Glorieux, PhD

Raymond Vanholder, MD, PhD*

Nephrology Section, Department of Internal Medicine and Pediatrics, Ghent University Hospital, Ghent, Belgium

*Corresponding author:

Raymond Vanholder

Ghent University Hospital

Nephrology Unit (entrance 12, route 1231)

Corneel Heymanslaan 10

900 Gent

Belgium

003293324511

Raymond.vanholder@ugent.be

Disclosure Statement: The authors have nothing to disclose

Keywords: (4-8 words to direct and optimize search results): uremic toxins, kidney damage,

cardiovascular damage; cardiorenal; pathophysiology 


\section{Key Points}

- Chronic Kidney Disease (CKD) increases cardiovascular risk, irrespective of its causes that could as such also induce cardiovascular damage.

- During CKD, several compounds are retained that are toxic to the cardio-vascular system.

- To reduce retained toxin concentration, currently alternatives to classical therapies such as dialysis and transplantation are considered, such as decreasing intestinal toxin generation. 


\section{Introduction}

Kidney and cardiac disease are interrelated. Both disorders share a number of common risk factors such as hypertension, and diabetes mellitus ${ }^{1}$. Kidney failure also is at the origin of risk factors and mechanisms that in turn cause cardiovascular complications, e.g. salt and fluid retention, hypertension and fluid overload. On the other hand cardiac failure or persistent arrhythmia lower the filtering capacity of the kidneys. Vascular damage linked to kidney disease itself, other factors, such as dyslipidemia, diabetes and hypertension, results in kidney hypoperfusion and decreased clearance, hence creating a vicious circle of heart and kidney affecting each other. This strong connection gave rise to the comprehensive term cardiorenal syndrome².

It has for long been thought that the cardiovascular damage of CKD was essentially the consequence of its predisposing risk factors, like diabetes. However, it has now been demonstrated that CKD causes cardiovascular damage independently from and superimposed upon these common risk factors ${ }^{3}$, just as it has also been shown that cardiovascular disease is related to end stage kidney disease (ESKD essentially on dialysis and transplantation) of kidney risk factors ${ }^{4}$. CKD as it develops is associated with a plethora of metabolic side-effects that can induce cardiovascular damage (fig 1). Those include chronic kidney disease - mineral bone disease (CKD-MBD), insulin resistance, thrombogenicity and inflammation as most important ones, hence often generating a vicious circle. The question then arises which factors linked to kidney dysfunction cause this progressive devastating disease.

The progression of CKD is linked to the gradual development of symptoms and complications (Table 1) associated with accumulation in the body of metabolites (uremic retention products) that in healthy people are excreted via the normal kidneys into the urine ${ }^{5}$. One of the most intensively studied aspects over the last decades in this area has been the role of these solutes in the deleterious effects of CKD, and especially, in cardiovascular damage. If retention solutes exert biological effects they are called uremic toxins, and the clinical picture that is caused is the uremic syndrome. The best known (but relatively inert) retained solutes are creatinine and urea. For the sake of clarity, creatinine and urea are only two of the many solutes associated with the uremic syndrome, which is attributable to a much larger array of solutes ${ }^{5}$ (discussed later). Most other solutes show a different pattern for generation, retention and removal by dialysis than creatinine and urea, which also implies that creatinine and urea concentration is only minimally representative of uremic toxicity. The term uremia is semantically linked to urea which is the most abundant retention compound, but this does not mean that urea is the only responsible compound, although urea exerts toxicity by itself ${ }^{6}$.

Because retention usually follows an exponential pattern, uremic toxin concentration increases faster as kidney function declines. Over the last decades, a large array of uremic compounds have been 
shown to exert biological and biochemical impacts. ${ }^{7}$ An increasing number of toxic uremic retention solutes have been identified. In this article we will review a selection of compounds with the most extensive proof of cardiovascular impact. This does not necessarily imply that these are also the most active cardiovascular toxins but at least they have emanated in highly convincing evidence after having most extensively been studied. Nevertheless, every decade still new uremic cardiovascular toxins are identified, still extending our pathophysiologic knowledge.

The picture that ensues is one of a complex pathophysiologic process, involving several solutes and mechanisms, and not simply related to just one or a few solutes, such as the classical retention products creatinine and urea. Uremic toxicity is also cardiovascular toxicity. As a consequence it is important to recognize CKD as well as AKI early enough, so as to enable the appropriate therapeutic measures to prevent its progression and complications, after consultation with a nephrologist or, if glomerular filtration rate (GFR) falls below $30 \mathrm{~mL} / \mathrm{min}$ ( $25 \%$ of normal), referral to a nephrologist.

\section{Effect of uremic toxins on the different cell types and pathophysiological pathways in the development of cardiovascular disease.}

The uremic toxins that are discussed in this review and their effect on the respective cells and processes are listed in table 2. Of note, there are more uremic cardiotoxins, but for reasons of text length we made a selection based on the amount of knowledge of negative cardiovascular effects of these toxins. For a more comprehensive review, the interested reader is referred to Vanholder et $\mathrm{al}^{7}$. Of note, increased concentration of several solutes during CKD is not only linked to decreased kidney clearance but also to a increase in generation, often in response to an increase in the concentration of other uremic toxins. This connection is especially the case for peptides such as $\beta_{2}$-microglobulin, parathyroid hormone, fibroblast growth factor-23 (FGF-23) and the cytokines (so-called middle molecules). Increased (intestinal) generation has also been suggested for the protein bound uremic toxins $^{8}$ (PBUTs), but this supposition was not confirmed in a recent study 9 . It is not always easy to delineate the relative contribution of clearance and generation in the solute increase due to CKD.

Even larger molecules which are not cleared by the kidneys (molecular weight too high to cross the glomerular filter, i.e. $\geq 58,000 D$ ) are increased in CKD in case of posttranslational modification of several compounds such as the lipoproteins ${ }^{10}$, which results in molecules that are more toxic than the genuine compounds. Although these are in strict sense no uremic toxins according to the current definition, their increased concentration is definitely also linked to uremic complications.

\section{Endothelial dysfunction}


Endothelial dysfunction (Fig. 2) is one of the leading causes in the development of atherosclerotic plaques and other vascular events. Severity of endothelial dysfunction is linked to the progression of CKD, meaning that patients in the more advanced stages of CKD are at higher risk of suffering from cardiovascular events caused by endothelial dysfunction. Chronic inflammation, increased production of reactive oxygen species (ROS) and decreased bioavailability of nitric oxide (NO) are main causes of endothelial dysfunction and can be the result of elevated plasma levels of uremic toxins. The uremic toxins that are best understood to induce endothelial dysfunction are discussed here.

The involvement of trimethylamine-N-oxide (TMAO) in the development of cardiovascular disease was first reported by Wang et al. in $2011^{11}$. TMAO is a product of oxidation by hepatic flavin monooxygenases of trimethylamine (TMA), a product of bacterial metabolisation of betaine, Lcarnitine and choline that are mainly found in nutrients of animal origin. Levels of TMAO were shown to be predictive of cardiovascular disease in a cohort of patients who suffered from cardiovascular events (myocardial infarction, stroke or death) when compared to an age- and gender-matched control group $^{11}$. In addition, dietary supplementation with TMAO or its precursors promoted experimental atherogenicity in mice. Studies in human umbilical vein endothelial cells (HUVECs) suggest endothelial dysfunction upon treatment with TMAO, as demonstrated by nucleotide-binding oligomerization domain-like receptor family pyrin domain-containing 3 (NLPR3) inflammasome activation which leads to increased expression of inflammatory cytokines, whereas increased production of ROS decreased NO production. ${ }^{12,13}$ Inhibition of metabolic production of TMA by the intestinal microbiota reduced TMAO plasma concentration and arterial damage in mice exposed to a load of TMAO precursors ${ }^{14}$.

Asymmetric dimethylarginine (ADMA), an inhibitor of endothelial nitric oxide synthase (eNOS) reduces functional levels of $\mathrm{NO}^{15}$. The combination of ADMA and indoxyl sulfate (IXS) is involved in the increased expression in aortic cells of senescence molecules, such as senescence-associated betagalactosidase (SA- $\beta$-gal). ${ }^{16}$

Urea, a product of protein metabolism, at physiologically relevant levels, increases ROS production in endothelial progenitor cells (EPCs) ${ }^{17}$. In addition, urea also decreases the number of EPCs and induces senescence in remaining $\mathrm{EPCs}^{17}$.

Association with increased mortality and major cardiovascular events in patients with CKD has repeatedly been reported for the colon-derived protein bound uremic toxins, indole-3 acetic acid (IAA), IXS and para-cresyl sulfate (pCS) $)^{18}$. IXS and pCS increase ROS production in HUVECS $)^{19,20}$. Besides increased ROS production, IXS also decreases eNOS, thereby decreasing production and bioavailability of $\mathrm{NO}^{21}$. In a rat intravital microscopic model, IXS caused shedding of heparan sulfate, pointing to disruption of the glycocalyx which could have played a role in the IxS-induced intravascular blood flow 
stagnation observed in the same study ${ }^{22}$. pCS, on the other hand, induced shedding of endothelial microparticles, that impair the NO signaling pathway ${ }^{23}$. An in vitro study of IAA in cultured human endothelial cells showed that IAA increases expression of the aryl hydrocarbon receptor (AhR) and subsequent production of cyclooxygenase 2 (COX-2-. In addition, production of ROS is linked to increased expression of the genes of interleukin (IL-) 6 and 8 , intracellular adhesion molecule 1 (ICAM1) and monocyte chemoattractant protein-1 (MCP-1) 18 , all of which are related to inflammation. Increased levels of all-caused mortality and CV events in patients with CKD. ${ }^{18}$

Chronic local inflammatory activity, characterized by increased expression of inflammatory cytokines is often induced by the accumulation of specific uremic toxins and plays an important role in the development of cardiovascular events in CKD patients. Tumor necrosis factor alpha (TNF- $\alpha$ ) was already proven to cause endothelial dysfunction in a time- and dose-dependent manner in bovine pulmonary arterial endothelial (CPAE) cells the early $1990 \mathrm{~s}^{24}$. This causative effect was later ascribed to increased oxidative stress due to increased ROS production and decreased NO availability ${ }^{21}$.

Taken together, endothelial dysfunction in CKD can be explained by multiple processes, among which are inflammation, oxidative stress and decreased NO availability, caused by direct and indirect effects of increased levels of uremic toxins (see Fig. 2).

\section{Vascular smooth muscle cells}

In CKD, both disturbances of the mineral balance and increased levels of uremic toxins affect vascular smooth muscle cell (VSMC) function resulting in changes in cell proliferation, migration, senescence and calcification, disturbing the cardiovascular system. Calcification of vSMCs can take place in conditions with increased levels of phosphorus and calcium ${ }^{25}$. After this transformation, cells show a more osteoblast-like phenotype and lose their contractility. This calcification results in thickening and stiffening of blood vessel walls.

vSMCs cultured in the presence of serum from CKD patients (10\%) show a significantly higher degree of proliferation and decreased expression of markers for the contractile phenotype of vSMCs, such as SM alpha actin, SM22 alpha, calponin and muscle myosin heavy chain, as compared to cells cultured in the presence of healthy serum $(10 \%)^{26}$.

In vitro, exposure to IXS leads to acute increased proliferation, migration, calcification and cell senescence in vSMCs. In these processes, there is an important role for the increased production of ROS induced by IXS, but also the changes in expression of platelet-derived growth factor receptor beta (PDGF $\beta-R)$ and other proteins are associated with the increased proliferation, as summarized by Hénaut et al. ${ }^{27}$. The proliferative effect of IxS is attributed to the activation of the p42/44 mitogen- 
activated protein kinase (MAPK)- and Aryl hydrocarbon Receptor (AhR)/ nuclear factor (NF)-KB pathway ${ }^{28,29}$. Besides IXS, the MAPK pathway can be activated by multiple other stimuli, among which growth factors, angiotensin II and erythropoietin. These effects were not seen in long-term exposure to lower doses of IxS. Decreased proliferation seen as chronic effect of exposure to IxS is believed to be due to upregulation of p21 and p27 which are cell cycle inhibitors ${ }^{30}$.

Hénaut et al. also report the effects of pCS on vSMC migration and apoptosis ${ }^{27}$. Here, migration is inhibited through upregulation of metalloproteinase (MMP) 2 and 9 and downregulation of tissue inhibitor of metalloproteinase (TIMP) 1 and $2^{31}$. pCS induces apoptosis of vSMCs by decreasing expression of the B-cell lymphoma 2 ( $\mathrm{Bcl} 2$ ) gene and increasing expression of $\mathrm{Bcl}-2$ associated $\mathrm{X}$ protein (Bax) gene, thereby initiating the cellular self-death signal ${ }^{27}$.

In addition, TNF- $\alpha$, IL-1 $\beta$ and IL- 6 all induce vSMC proliferation, migration, apoptosis and calcification. TNF- $\alpha$ exerts its effect by increasing ROS production and altering the expression of TNF-related apoptosis-inducing ligand (TRAIL), PDGF $\beta$, MMP 2 and 9 , caspases 3 and 8 and Klotho ${ }^{27}$. IL-1 $\beta$ is involved in increased VSMC proliferation, migration, apoptosis and calcification via altered expression of many of the previously mentioned proteins. IL-1 $\beta$ also increased expression of purinergic P2Y2 receptor (P2Y2R); receptor for advanced glycation end products (RAGE) and high mobility group box 1 $(\text { HMGB1 })^{27}$. This leads to proliferation, increased production of apoptosis anigen 1 (APO-1) and NO, leading to apoptosis and induction of tissue non-specific alkaline phosphatase (TNAP) resulting in calcification of vSMCs. IL- 6 mostly mediates the effects of TNF- $\alpha$, but also exerts its own effects by increasing actin polymerization and expression of phospho-focal adhesion kinase (pFAK)/ paxillin, signal transducer and activator of transcription 1 (Stat1), heat shock protein (HSP)70 and receptor activatory of nuclear factor kappa-B ligand (RANKL) ${ }^{27}$.

Each of the different effects of uremic toxins on vSMCs as well as their combination lead to increased risk for cardiovascular disease.

\section{Leukocyte activation and adhesion}

Leukocyte activation and adhesion to endothelium is part of the normal inflammatory process. In CKD, chronic inflammation leads to over-activation and adhesion of leukocytes which can act as an initial stage of sclerotic plaque formation or vascular stiffening. In normal conditions, the activation of adhesion molecules, like vascular and intracellular cell adhesion molecules-1 (VCAM-1 and ICAM-1) and E-selectin, which lead to recruitment of macrophages and monocytes, is tightly regulated via the NF-KB pathway. In CKD, the activation of this pathway is increased due to direct exposure to higher levels of uremic toxins or by other inflammatory processes. Long-term activation of the adhesion 
molecules can lead to collection and pooling of immune cells in the blood vessels, leading to the development of atherosclerotic plaques and increased risk of cardiovascular events.

TMAO and symmetric dimethylarginine (SDMA) are among the uremic toxins that affect the activation and adhesion of leukocytes. They both increase macrophage migration and expression of ICAM-1 via upregulation of TNF- $\alpha$ and IL- $6^{32,33}$. On top of that, increased levels of IL-6 reduce NO availability, by inhibition of eNOS ${ }^{34}$, which prolongs the inflammation reaction ${ }^{35}$.

Among the PBUTs, an effect on leukocyte recruitment is seen for IXS, pCS and para-cresyl glucuronide (pCG), whereby presence of pCS resulted in an increased number of rolling leukocytes, and the combination of pCG and pCS caused albumin leakage trough the vessel wall and impaired intravascular blood flow. ${ }^{22}$

Treatment of HUVECs met IXS increased the expression of E-selectin, but not that of ICAM-1 or VCAM1. THP-1 (human monocytic cell lin) adhesion was induced upon stimulation with IxS by activation of the c-JUN N-terminal kinase (JNK), p38 MAPK, and NF-KB pathways ${ }^{36}$.

Overactivation of leukocytes causes chronic inflammation in CKD and vice versa. This inflammation induces increased adhesion to the vessel wall and subsequently leads to increased CV damage, resulting in atherosclerosis, left ventricular hypertrophy, systolic cardiac dsyfunction, progression of kidney failure, and higher mortality. This chronic inflammatory state is increasingly present as CKD progresses and is most prominent in patients undergoing dialysis ${ }^{37}$.

\section{Chronic kidney disease and mineral bone disease (CKD-MBD)}

Mineral bone disease (MBD) is a common complication of CKD, due to the disturbed balance in mineral homeostasis, mainly that of calcium and phosphorus. MBD does not only affect the skeletal system, but can also affect the vasculature by means of abnormal mineral disposition. Disposition of calcium salts can occur in both the intimal and medial layers of arteries or in the heart valves ${ }^{38}$. A major uremic toxin that is associated with the development of MBD in CKD patients is fibroblast growth factor (FGF)23. Its primary role is regulation and maintenance of phosphate homeostasis. In advanced stages of CKD, levels of FGF-23 can increase up to a 1000-fold, due to the development of FGF-23 resistance ${ }^{39}$. Increased levels of FGF-23 in patients with CKD are associated with development of cardiovascular disease, specifically vascular damage and left ventricular hypertrophy ${ }^{40}$. In 2011, Faul et al. showed that FGF-23 causes cardiac hypertrophy in rat cardiomyocytes via activation of the FGF receptordependent activation of the calcineurin- nuclear factor of activated T cell (NFAT) ${ }^{41}$.

The exact mechanisms by which gut derived uremic toxins lead to MBD are not fully understood, but there is a direct correlation between levels of pCS and IXS and decreased bone-density levels and other 
bone abnormalities ${ }^{42}$. Direct effects of IxS on bone formation have been found in in vitro experiments with mouse osteoblasts. In these cells, IXS suppressed the expression of genes related to bone formation (osterix, osteocalcin and bone morphogenetic protein-2 (BMP-2)) ${ }^{43}$. Exposure to $\mathrm{pCS}$ showed that pCS decreased parathyroid hormone (PTH)-induced cAMP-activation and increased ROS production in mouse osteoblasts. It also led to increased activation of the JNK-p23 MAPK pathway thereby inducing cellular dysfunction, increased DNA fragmentation and reduced osteoblast cell proliferation ${ }^{44}$.

\section{Insulin resistance}

Insulin resistance (IR) is common in patients with advanced stages of CKD and in ESKD. Decreased insulin sensitivity gives rise to increased levels of insulin, associated with increased risk for the development of atherosclerosis ${ }^{45}$.

Hyperuricemia has been demonstrated to be strongly associated with IR and other forms of abnormal glucose metabolism. It is believed that IR in CKD is caused by activation of E3 ubiquitin ligases, that bind to insulin receptor substrate-1 (IRS-1 $)^{46}$. Subsequently, IRS-1 is degraded which suppresses insulininduced intracellular signaling, leading to IR.

Increased urea, at levels measured in advanced stages of CKD, induces the production of ROS, causing modulation of O-linked $\beta$ - $\mathrm{N}$-acethylglucosamine (O-GlcNAc), and reducing insulin stimulated IRS thyrosine phosphorylation, PKB-Akt phosphorylation and glucose transport, which all together lead to insulin resistance ${ }^{47}$.

ADMA is also associated with the onset of IR. Studies in transgenic mice, overexpressing dimethylaminohydrolase (DDAH-I), which decreases intracellular concentration of methylarginines among which ADMA, show increased insulin sensitivity compared to mice not expressing DDAH-1 ${ }^{48}$. This finding suggests ADMA is responsible for decreased insulin sensitivity in these mice.

pCS induces IR by altering the signaling in skeletal muscle through ERK1/2 activation, which affects the phosphorylation of PKB/Akt ${ }^{49}$. Treatment with an ERK1/2 inhibitor (U0126) restored the insulininduced phosphorylation of PKB/Akt.

Of the cytokines previously discussed, there is a definite role for IL- 6 and a potential role for other inflammatory cytokines in the production of suppressor of cytokine signaling 3 (SOCS 3). Production of SOCS3 will reduce the levels of IRS-1 and thereby reduce insulin-induced intracellular signaling, as seen in hyperuricemia ${ }^{50}$. The development of IR in patients with CKD will lead to increased risk of other (cardiovascular) effects, such as hypertension, oxidative stress, endothelial dysfunction, dyslipidemia and type 2 diabetes, increase the risk of cardiovascular events ${ }^{51}$. 


\section{Thrombogenicity}

Patients suffering from CKD have increased risk of thrombosis-related complications, as they have increased serum levels of tissue factor (TF), fibrinogen and D-dimer ${ }^{52-54}$. The exact mechanisms that lead to increased risk of thrombosis are not fully understood, but the role of individual uremic toxins in relation to thrombosis has been studied, and there is a strong association between IXS and IAA and the pro-thrombotic state. They both activate the aryl hydrocarbon receptor (AhR) which stimulates the production and release of TF in HUVECs ${ }^{55}$. Increased thrombogenicity is also seen in a rat model conform with advanced $C K D^{56}$. Uremic solutes, IAA, IXS, but also uric acid, also affect the production of TF by vSMCs ${ }^{56}$. In addition, they stabilized TF (longer half-life) and decreased ubiquitination. Activation of the AhR induces cyclooxygenase (COX)2 expression via the p38MAPK/NF-KB pathway. The increase in COX2 enhances the synthesis of prostaglandin E2 (PGE2) which promotes thrombosis by binding to the platelet EP3 receptor and inducing platelet aggregation ${ }^{57}$. The exact mechanism by which uric acid is involved in regulation of TF is yet to be not clear, and several direct and indirect mechanisms have been hypothesized, such as an effect on translation, activity changing interaction with other clotting factors of the inflammatory pathway and activation of the inflammatory pathway ${ }^{56}$. Increased thrombogenicity is also associated with increased kynurenine in CKD-patients. Kolachalama et al. proved that kynurenine acts via an AhR-dependent mechanism to increase thrombosis after vascular injury in animal models ${ }^{58}$.

\section{Discussion}

The links between kidney and cardiovascular disease are multiple, leading to a snowball effect as both conditions progress. In this publication, we summarize the deleterious impact of uremic toxins on the cardiovascular system. A complex picture ensues, involving multiple systemic mechanisms (see fig 1), pathways and toxins (see fig 2, table 2).

Inflammation, oxidative stress and decreased NO availability are the main factors that act on and disturb cellular processes in CKD. Loss of endothelial function, VSMC calcification, leukocyte activation, CKD-MBD, IR and increased thrombogenicity are all induced by increased levels of one or more uremic toxins that are accumulated in the body due to impaired kidney excretion and/or increased production. Loss of endothelial function forms the basis of the development of vascular damage, and it is reported that the risk of servere CV events caused by endothelial dysfunction increases from $140 \%$ in CKD stage 3 up to $340 \%$ in $\mathrm{ESRD}^{59}$. Likewise, it is reported that $49 \%$ of patients with CKD stage 3 have coronary 
artery disease, versus only $19 \%$ of patients with normal kidney function. Consequences of vascular calcification include vessel stiffness and loss of elasticity leading to lower oxygen delivery to the body tissues, worsening of endothelial dysfunction and increased blood pressure causing left ventricular hypertrophy ${ }^{45}$.

In view of the cardiovascular impact of uremic toxins, it is relevant to decrease their concentration. The principal questions in this context are: 1) which molecules are the most appropriate candidates to be removed; 2) which therapeutic options are available to reach this aim? Based on clinical and experimental evidence, the authors previously made a ranking of the solutes with the most deleterious toxic effects ${ }^{7}$ (table 3). This ranking refers to a broad array of toxic effects, but, since most of them directly or indirectly affect the cardiovascular system, this list is also applicable for cardiovascular toxicity. These might be the solutes that should be most focused on.

Unfortunately, few therapeutic intervention are directly designed to decrease specific uremic toxins and none of these resulted in convincing outcome advantages ${ }^{60}$. Probably, decreasing one single toxin is not sufficient to improve outcomes, in view of the broad array of solutes and mechanisms involved in the uremic toxicity.

The best known options for overall (unspecific) toxin removal are dialysis and transplantation (commonly referred to as renal replacement therapy - RRT), which are part of the therapeutic options at the disposal of nephrologists. Perhaps one of the most convincing arguments in favor of a therapeutic outcome benefit is observed for hemodialysis or hemodiafiltration (a combination between dialysis and ultrafiltration) with large pore (high-flux) hemodialysis membranes, although also these are based on observational or secondary outcome analyses ${ }^{61-63}$. Larger pore membranes remove better than other membranes larger retention solutes (so-called middle molecules), which also outnumber other types of retention solutes in our toxicity ranking (table 3).

Because RRT only is applied at more advanced stages of CKD, earlier lowering of uremic cardiotoxins can only be made possible by alternative non-extracorporeal therapeutic options, such as refraining progression of kidney disease, which can be elicited by primary and secondary prevention, which both will be discussed in other sections of this issue. Another option, especially for PBUTs, is decreasing intestinal generation ${ }^{64,65}$. As amino acids are the precursors of most of these toxins, a logical approach would be to reduce dietary protein intake, which indeed has been linked to slowing down progression of kidney disease, although the available evidence was considered of low quality ${ }^{66}$. However, the negative side of this intervention is the risk to worsen existing malnutrition or systemic protein depletion, especially in frail patients and those with heavy proteinuria, in which case protein restriction 
might be less beneficial. In general, in CKD not on dialysis or shortly $(3 \mathrm{~m})$ after transplantation, a protein intake of $0.6-0.8 \mathrm{~g} / \mathrm{kg} /$ day is recommended ${ }^{67}$.

Modifying the composition or function of intestinal microbiota is another interesting option. Probiotics (changing intestinal microbiota composition), prebiotics (changing function of intestinal microbiota) and synbiotics (combining both) all have the potential to modify intestinal microbiota favorably towards less production of precursors of PBUTs. Even if recent data suggest that, contrary to the previously held belief ${ }^{8}$, intestinal generation of PBUTs does not change as CKD becomes more severe ${ }^{9}$, it still remains worthwhile to decrease intestinal production rate, to diminish toxic impact on the kidneys and the cardiovascular system. Controlled studies on the effect of these xenobiotics are scarce and hard outcome studies are missing entirely ${ }^{68}$. Available data suggests that not all CKD patients react in the same way to the same intervention ${ }^{69,70}$, and that not all interventions have the same effect ${ }^{8}$. Probably, one size does not fit all, and our knowledge of intestinal toxin generation is still too fragmentary to allow developing a definite therapeutic roadmap. In addition, as not all PBUTs seem to be generated by the same microbiota ${ }^{71}$, it is unlikely that one solution will suffice to decrease the whole array of PBUTs. Further study is needed to allow a better targeted approach. By default, we suggest to focus especially on $p$-cresyl sulfate, of which the intestinally generated mother compound, p-cresol, is deleterious at the site of origin (the intestine), whereas the increased plasma levels of, $p$-cresyl sulfate by current knowledge are assumed to play a central role in overall and CV toxicity ${ }^{9,72}$.

The other option to decrease body uptake of precursors of PBUTs is peroral administration of sorbents that are active inside the intestinal lumen, such as AST-120 (Kremezin $\left.{ }^{\mathrm{R}}\right)^{73}$. Randomized controlled trials did however not confirm the hypothesized impact on progression of $\mathrm{CKD}^{74,75}$.

In conclusion, the cardiovascular system and the kidneys, when they are diseased, show a complex interaction that is deleterious to both. In this publication, we describe a selection of uremic toxins with the most abundant evidence of negative cardiovascular impact. It seems logical that decreasing the concentration of these solutes will attenuate this effect. With the current therapeutic resources, primary and secondary prevention measures are the most effective means to reach these aims. However, therapeutic approaches to kidney disease have remained almost unchanged since more than half a century and novel therapeutic paradigms, spanning both pharmacological and technological approaches are urgently needed ${ }^{76}$. Such measures will not only benefit kidney patients but also a large proportion of people with cardiovascular disease, in view of the close links between both organ systems and their frequent interaction. 


\section{Summary}

The links between kidney disease and CVD are multiple, leading to a snowball effect as both conditions progress. This article summarizes the deleterious impact of uremic toxins on the CV system. A complex picture ensues, involving multiple systemic mechanisms, pathways, and toxins. Inflammation, oxidative stress, and decreased NO availability are the main factors that act on and disturb cellular processes in CKD. Loss of endothelial function, vSMC proliferation and calcification, leukocyte activation, CKD-MBD, IR, and increased thrombogenicity are all induced by increased levels of one or more uremic toxins that are accumulated in the body because of impaired kidney excretion and/or increased production.

\section{Clinics Care Points}

- Prevention of development and progression of CKD by lifestyle measures and pharmacologic intervention are the most appropriate ways to forestall CKD-induced CVD, especially if this approach is implemented before or in the early stages of CKD.

- Reduction of dietary protein intake may prevent progression of CKD but should be applied carefully to avoid malnutrition. Ideally, normal protein intake $(0.6-0.8 \mathrm{~g} / \mathrm{kg}$ body weight $/ \mathrm{d})$ is indicated; however, in hemodialysis patients, protein intake should be increased because of protein losses caused by the dialysis procedure.

- Modifying the function and/or composition of intestinal microbiota by prebiotics probiotics, or synbiotics may be another option to decrease the concentration of uremic cardiotoxins, but this approach needs further study.

- In ESRD, hemodialysis or hemodiafiltration using large-pore dialyzer membranes, are likely to improve CV outcomes, especially in high-risk patients 


\section{References}

1. Said S, Hernandez GT. The link between chronic kidney disease and cardiovascular disease. J Nephropathol. 2014;3(3):99-104.

2. Rangaswami J, Bhalla V, Blair JEA, et al. Cardiorenal Syndrome: Classification, Pathophysiology, Diagnosis, and Treatment Strategies: A Scientific Statement From the American Heart Association. Circulation. 2019;139(16):e840-e878.

3. Wan EYF, Yu EYT, Chin WY, et al. Burden of CKD and Cardiovascular Disease on Life Expectancy and Health Service Utilization: a Cohort Study of Hong Kong Chinese Hypertensive Patients. Journal of the American Society of Nephrology : JASN. 2019;30(10):1991-1999.

4. Ishigami J, Cowan LT, Demmer RT, et al. Incident Hospitalization with Major Cardiovascular Diseases and Subsequent Risk of ESKD: Implications for Cardiorenal Syndrome. Journal of the American Society of Nephrology : JASN. 2020;31(2):405-414.

5. Vanholder R, De Smet R, Glorieux G, et al. Review on uremic toxins: classification, concentration, and interindividual variability. Kidney international. 2003;63(5):1934-1943.

6. Vanholder R, Gryp T, Glorieux G. Urea and chronic kidney disease: the comeback of the century? (in uraemia research). Nephrology, dialysis, transplantation : official publication of the European Dialysis and Transplant Association - European Renal Association. 2017.

7. Vanholder R, Pletinck A, Schepers E, Glorieux G. Biochemical and Clinical Impact of Organic Uremic Retention Solutes: A Comprehensive Update. Toxins. 2018;10(1).

8. Vanholder R, Pletinck A, Schepers E, Glorieux G. Biochemical and Clinical Impact of Organic Uremic Retention Solutes: A Comprehensive Update. Toxins (Basel). 2018;10(1):33.

9. Gryp T, De Paepe K, Vanholder R, et al. Gut microbiota generation of protein-bound uremic toxins and related metabolites is not altered at different stages of chronic kidney disease. Kidney international. 2020;97(6):1230-1242.

10. Speer T, Rohrer L, Blyszczuk $P$, et al. Abnormal high-density lipoprotein induces endothelial dysfunction via activation of Toll-like receptor-2. Immunity. 2013;38(4):754-768.

11. Wang Z, Klipfell E, Bennett BJ, et al. Gut flora metabolism of phosphatidylcholine promotes cardiovascular disease. Nature. 2011;472(7341):57-63.

12. Chen ML, Zhu XH, Ran L, Lang HD, Yi L, Mi MT. Trimethylamine-N-Oxide Induces Vascular Inflammation by Activating the NLRP3 Inflammasome Through the SIRT3-SOD2-mtROS Signaling Pathway. Journal of the American Heart Association. 2017;6(9).

13. Sun $\mathrm{X}$, Jiao $\mathrm{X}, \mathrm{Ma} \mathrm{Y}$, et al. Trimethylamine $\mathrm{N}$-oxide induces inflammation and endothelial dysfunction in human umbilical vein endothelial cells via activating ROS-TXNIP-NLRP3 inflammasome. Biochemical and biophysical research communications. 2016;481(1-2):63-70.

14. Wang Z, Roberts AB, Buffa JA, et al. Non-lethal Inhibition of Gut Microbial Trimethylamine Production for the Treatment of Atherosclerosis. Cell. 2015;163(7):1585-1595.

15. Vallance $\mathrm{P}$, Leone A, Calver A, Collier J, Moncada S. Accumulation of an endogenous inhibitor of nitric oxide synthesis in chronic renal failure. Lancet. 1992;339(8793):572-575.

16. Adelibieke Y, Shimizu H, Muteliefu G, Bolati D, Niwa T. Indoxyl sulfate induces endothelial cell senescence by increasing reactive oxygen species production and p53 activity. Journal of renal nutrition : the official journal of the Council on Renal Nutrition of the National Kidney Foundation. 2012;22(1):86-89.

17. Giardino I, D'Apolito M, Brownlee M, et al. Vascular toxicity of urea, a new "old player" in the pathogenesis of chronic renal failure induced cardiovascular diseases. Turk Pediatri Ars. 2017;52(4):187-193.

18. Dou L, Sallée M, Cerini C, et al. The cardiovascular effect of the uremic solute indole-3 acetic acid. J Am Soc Nephrol. 2015;26(4):876-887.

19. Watanabe $\mathrm{H}$, Miyamoto $\mathrm{Y}$, Enoki $\mathrm{Y}$, et al. $\mathrm{p}$-Cresyl sulfate, a uremic toxin, causes vascular endothelial and smooth muscle cell damages by inducing oxidative stress. Pharmacology research \& perspectives. 2015;3(1):e00092. 
20. Yu M, Kim YJ, Kang DH. Indoxyl sulfate-induced endothelial dysfunction in patients with chronic kidney disease via an induction of oxidative stress. Clin J Am Soc Nephrol. 2011;6(1):30-39.

21. Agnoletti L, Curello S, Bachetti T, et al. Serum from patients with severe heart failure downregulates eNOS and is proapoptotic: role of tumor necrosis factor-alpha. Circulation. 1999;100(19):1983-1991.

22. Pletinck A, Glorieux G, Schepers E, et al. Protein-bound uremic toxins stimulate crosstalk between leukocytes and vessel wall. Journal of the American Society of Nephrology : JASN. 2013;24(12):1981-1994.

23. Meijers BKI, Van kerckhoven S, Verbeke K, et al. The Uremic Retention Solute p-Cresyl Sulfate and Markers of Endothelial Damage. American Journal of Kidney Diseases. 2009;54(5):891901.

24. Goldblum SE, Sun WL. Tumor necrosis factor-alpha augments pulmonary arterial transendothelial albumin flux in vitro. The American journal of physiology. 1990;258(2 Pt 1):L57-67.

25. Cozzolino M, Ciceri P, Galassi A, et al. The Key Role of Phosphate on Vascular Calcification. Toxins (Basel). 2019;11(4):213.

26. Monroy MA, Fang J, Li S, et al. Chronic kidney disease alters vascular smooth muscle cell phenotype. Front Biosci (Landmark Ed). 2015;20:784-795.

27. Hénaut L, Mary A, Chillon J-M, Kamel S, Massy ZA. The Impact of Uremic Toxins on Vascular Smooth Muscle Cell Function. Toxins (Basel). 2018;10(6):218.

28. Yamamoto $\mathrm{H}$, Tsuruoka S, loka $\mathrm{T}$, et al. Indoxyl sulfate stimulates proliferation of rat vascular smooth muscle cells. Kidney international. 2006;69(10):1780-1785.

29. Ng HY, Bolati W, Lee CT, et al. Indoxyl Sulfate Downregulates Mas Receptor via Aryl Hydrocarbon Receptor/Nuclear Factor-kappa B, and Induces Cell Proliferation and Tissue Factor Expression in Vascular Smooth Muscle Cells. Nephron. 2016;133(3):205-212.

30. Mozar $A$, Louvet $L$, Morlière $P$, et al. Uremic toxin indoxyl sulfate inhibits human vascular smooth muscle cell proliferation. Therapeutic apheresis and dialysis : official peer-reviewed journal of the International Society for Apheresis, the Japanese Society for Apheresis, the Japanese Society for Dialysis Therapy. 2011;15(2):135-139.

31. Han H, Chen $\mathrm{Y}$, Zhu Z, et al. p-Cresyl sulfate promotes the formation of atherosclerotic lesions and induces plaque instability by targeting vascular smooth muscle cells. Frontiers of medicine. 2016;10(3):320-329.

32. Geng J, Yang C, Wang B, et al. Trimethylamine N-oxide promotes atherosclerosis via CD36dependent MAPK/JNK pathway. Biomedicine \& pharmacotherapy = Biomedecine \& pharmacotherapie. 2018;97:941-947.

33. Schepers E, Barreto DV, Liabeuf S, et al. Symmetric dimethylarginine as a proinflammatory agent in chronic kidney disease. Clin J Am Soc Nephrol. 2011;6(10):2374-2383.

34. Hung MJ, Cherng WJ, Hung MY, Wu HT, Pang JH. Interleukin-6 inhibits endothelial nitric oxide synthase activation and increases endothelial nitric oxide synthase binding to stabilized caveolin-1 in human vascular endothelial cells. Journal of hypertension. 2010;28(5):940-951.

35. Didion SP. Cellular and Oxidative Mechanisms Associated with Interleukin-6 Signaling in the Vasculature. Int J Mol Sci. 2017;18(12):2563.

36. Ito $S$, Osaka M, Higuchi Y, Nishijima F, Ishii H, Yoshida M. Indoxyl sulfate induces leukocyteendothelial interactions through up-regulation of E-selectin. J Biol Chem. 2010;285(50):38869-38875.

37. Nowak KL, Chonchol M. Does inflammation affect outcomes in dialysis patients? Semin Dial. 2018;31(4):388-397.

38. Valdivielso JM. Vascular calcification: types and mechanisms. Nefrología (English Edition). 2011;31(2):142-147. 
39. Galitzer H, Ben-Dov IZ, Silver J, Naveh-Many T. Parathyroid cell resistance to fibroblast growth factor 23 in secondary hyperparathyroidism of chronic kidney disease. Kidney international. 2010;77(3):211-218.

40. Russo D, Battaglia Y. Clinical Significance of FGF-23 in Patients with CKD. Int J Nephrol. 2011;2011:364890-364890.

41. Faul C, Amaral AP, Oskouei B, et al. FGF23 induces left ventricular hypertrophy. The Journal of clinical investigation. 2011;121(11):4393-4408.

42. Black AP, Cardozo LFMF, Mafra D. Effects of Uremic Toxins from the Gut Microbiota on Bone: A Brief Look at Chronic Kidney Disease. Therapeutic Apheresis and Dialysis. 2015;19(5):436440.

43. Watanabe $\mathrm{K}$, Tominari $\mathrm{T}$, Hirata $\mathrm{M}$, et al. Indoxyl sulfate, a uremic toxin in chronic kidney disease, suppresses both bone formation and bone resorption. FEBS Open Bio. 2017;7(8):1178-1185.

44. Tanaka $\mathrm{H}$, Iwasaki $\mathrm{Y}$, Yamato $\mathrm{H}$, et al. p-Cresyl sulfate induces osteoblast dysfunction through activating JNK and p38 MAPK pathways. Bone. 2013;56(2):347-354.

45. Capusa C, Popescu D. Mechanisms and clinical implications of vascular calcifications in chronic kidney disease. In: Rath T, editor. Chronic kidney disease, from pathophysiology to clinical improvements. London: Intechopen; 2017. p. 61-82.

46. Thomas SS, Zhang L, Mitch WE. Molecular mechanisms of insulin resistance in chronic kidney disease. Kidney international. 2015;88(6):1233-1239.

47. D'Apolito M, Du X, Zong H, et al. Urea-induced ROS generation causes insulin resistance in mice with chronic renal failure. The Journal of clinical investigation. 2014;124(10):1-1.

48. Sydow K, Mondon CE, Schrader J, Konishi H, Cooke JP. Dimethylarginine dimethylaminohydrolase overexpression enhances insulin sensitivity. Arteriosclerosis, thrombosis, and vascular biology. 2008;28(4):692-697.

49. Koppe L, Pelletier CC, Alix PM, et al. Insulin resistance in chronic kidney disease: new lessons from experimental models. Nephrology Dialysis Transplantation. 2014;29(9):1666-1674.

50. Zhang L, Du J, Hu Z, et al. IL-6 and Serum Amyloid A Synergy Mediates Angiotensin II-Induced Muscle Wasting. Journal of the American Society of Nephrology. 2009;20(3):604.

51. Tangvarasittichai $S$. Oxidative stress, insulin resistance, dyslipidemia and type 2 diabetes mellitus. World journal of diabetes. 2015;6(3):456-480.

52. Mercier E, Branger B, Vecina F, et al. Tissue factor coagulation pathway and blood cells activation state in renal insufficiency. The hematology journal : the official journal of the European Haematology Association. 2001;2(1):18-25.

53. Nunns GR, Moore EE, Chapman MP, et al. The hypercoagulability paradox of chronic kidney disease: The role of fibrinogen. Am J Surg. 2017;214(6):1215-1218.

54. Karami-Djurabi R, Klok FA, Kooiman J, Velthuis SI, Nijkeuter M, Huisman MV. D-dimer Testing in Patients with Suspected Pulmonary Embolism and Impaired Renal Function. The American Journal of Medicine. 2009;122(11):1050-1053.

55. Gondouin B, Cerini C, Dou L, et al. Indolic uremic solutes increase tissue factor production in endothelial cells by the aryl hydrocarbon receptor pathway. Kidney international. 2013;84(4):733-744.

56. Chitalia VC, Shivanna S, Martorell J, et al. Uremic serum and solutes increase post-vascular interventional thrombotic risk through altered stability of smooth muscle cell tissue factor. Circulation. 2013;127(3):365-376.

57. Addi T, Dou L, Burtey S. Tryptophan-Derived Uremic Toxins and Thrombosis in Chronic Kidney Disease. Toxins (Basel). 2018;10(10):412.

58. Kolachalama VB, Shashar M, Alousi F, et al. Uremic Solute-Aryl Hydrocarbon Receptor-Tissue Factor Axis Associates with Thrombosis after Vascular Injury in Humans. Journal of the American Society of Nephrology. 2018;29(3):1063-1072.

59. Roumeliotis S, Mallamaci F, Zoccali C. Endothelial Dysfunction in Chronic Kidney Disease, from Biology to Clinical Outcomes: A 2020 Update. J Clin Med. 2020;9(8):2359. 
60. Vanholder R, Van Laecke S, Glorieux G, Verbeke F, Castillo-Rodriguez E, Ortiz A. Deleting Death and Dialysis: Conservative Care of Cardio-Vascular Risk and Kidney Function Loss in Chronic Kidney Disease (CKD). Toxins. 2018;10(6).

61. Locatelli F, Martin-Malo A, Hannedouche T, et al. Effect of membrane permeability on survival of hemodialysis patients. Journal of the American Society of Nephrology : JASN. 2009;20(3):645-654.

62. Nistor I, Palmer SC, Craig JC, et al. Convective versus diffusive dialysis therapies for chronic kidney failure: an updated systematic review of randomized controlled trials. American journal of kidney diseases : the official journal of the National Kidney Foundation. 2014;63(6):954-967.

63. WeinhandI ED, Foley RN, Gilbertson DT, et al. Propensity-matched mortality comparison of Incident hemodialysis and peritoneal dialysis patients. J Am Soc Nephrol 2010;21(3):499.

64. Koppe L, Mafra D, Fouque D. Probiotics and chronic kidney disease. Kidney international. 2015;88(5):958-966.

65. Schepers E, Glorieux G, Vanholder R. The gut: the forgotten organ in uremia? Blood purification. 2010;29(2):130-136.

66. Fouque D, Laville M. Low protein diets for chronic kidney disease in non diabetic adults. The Cochrane database of systematic reviews. 2009(3):CD001892.

67. Vanholder R, Fouque D, Glorieux G, et al. Clinical management of the uraemic syndrome in chronic kidney disease. The lancet Diabetes \& endocrinology. 2016;4(4):360-373.

68. Rossi M, Klein K, Johnson DW, Campbell KL. Pre-, pro-, and synbiotics: do they have a role in reducing uremic toxins? A systematic review and meta-analysis. International journal of nephrology. 2012;2012:673631.

69. Meijers BK, De Preter V, Verbeke K, Vanrenterghem Y, Evenepoel P. p-Cresyl sulfate serum concentrations in haemodialysis patients are reduced by the prebiotic oligofructose-enriched inulin. Nephrology, dialysis, transplantation : official publication of the European Dialysis and Transplant Association - European Renal Association. 2010;25(1):219-224.

70. Rossi M, Johnson DW, Morrison M, et al. Synbiotics Easing Renal Failure by Improving Gut Microbiology (SYNERGY): A Randomized Trial. Clinical journal of the American Society of Nephrology : CJASN. 2016.

71. Joossens M, Faust K, Gryp T, et al. Gut microbiota dynamics and uraemic toxins: one size does not fit all. Gut. 2018.

72. Glorieux G, Vanholder R, Van Biesen W, et al. Free P-cresyl sulfate shows the highest association with cardiovascular outcome in chronic kidney disease. Nephrol Dial Transpl 2021. https://doi.org/10.1093/ ndt/gfab004.

73. Schulman G, Agarwal R, Acharya M, Berl T, Blumenthal S, Kopyt N. A multicenter, randomized, double-blind, placebo-controlled, dose-ranging study of AST-120 (Kremezin) in patients with moderate to severe CKD. American journal of kidney diseases : the official journal of the National Kidney Foundation. 2006;47(4):565-577.

74. Schulman G, Berl T, Beck GJ, et al. Randomized Placebo-Controlled EPPIC Trials of AST-120 in CKD. Journal of the American Society of Nephrology : JASN. 2015;26(7):1732-1746.

75. Cha RH, Kang SW, Park CW, et al. A Randomized, Controlled Trial of Oral Intestinal Sorbent AST-120 on Renal Function Deterioration in Patients with Advanced Renal Dysfunction. Clinical journal of the American Society of Nephrology : CJASN. 2016.

76. Zoccali C, Vanholder R, Wagner CA, et al. Funding kidney research as a public health priority: challenges and opportunities. Nephrology, dialysis, transplantation : official publication of the European Dialysis and Transplant Association - European Renal Association. 2020. 


\section{ACKNOWLEDGMENTS}

S. Valkenburg is an early stage researcher on a project that has received funding from the European Union's Horizon 2020 research and innovation programme under grant agreement No 860329 (STRATEGY-CKD). 


\section{Figure/Table Legends}

Figure 1: The interrelation between kidney damage and cardiovascular damage, risk factors and mechanisms involved. Arrows directed from cause to consequence. Issues in bold are discussed in detail in the text. Several mechanisms, especially those associated with inflammation and its consequeces, enhance each other. CKD-MBD, chronic kidney disease-mineral bone disorder.

Figure 2: Cellular mechanisms in endothelial dysfunction caused by the uremic toxins considered in this publication. The uremic toxins are transported from the plasma into the cytosol via different iontransporter proteins (CAT, OAT and OCT). Their intercellular effects can be divided into three major pathways: (1) Decrease of NO-bioavailability by inhibition of eNOS as demonstrated for ADMA and IXS, and emptying of NO-stores via ROS production as demonstrated for IXS, pCS and TMAO; (2) Activation of the NLPR3-inflammasome via nuclear NF-KB activation resulting in the generation of active inflammatory cytokines as demonstrated for TMAO; (3) Direct production of inflammatory cytokines and activation of Caspase- 1 via NF-KB activation and translocation to the nucleus as demonstrated for TMAO. Full arrows, facilitation; dashed arrow, transport/translocation; bar-headed arrow, inhibition. Abbreviations: ADMA, asymmetric dimethylarginine; IXS, indoxyl sulfate; pCS, paracresyl sulfate; TMAO, trimethylamine-N-oxide; CAT, cationic amino acid transporter; OAT, organic anion transporter; OCT, organic cation transporter; eNOS, endothelial nitric oxide synthase; NO, nitric oxide; ROS, reactive oxygen species; TRX, thioredoxin; TXNIP, thioredoxin interacting protein; Pro-Casp-1, pro-caspase-1; ACS, apoptosis associated speck-like protein; NLPR3, nod-like receptor family pyrin domain containing 3 ; NF-KB, nuclear factor $\mathrm{KB}$; IKB, inhibitor of NF-KB. 
Figure 1

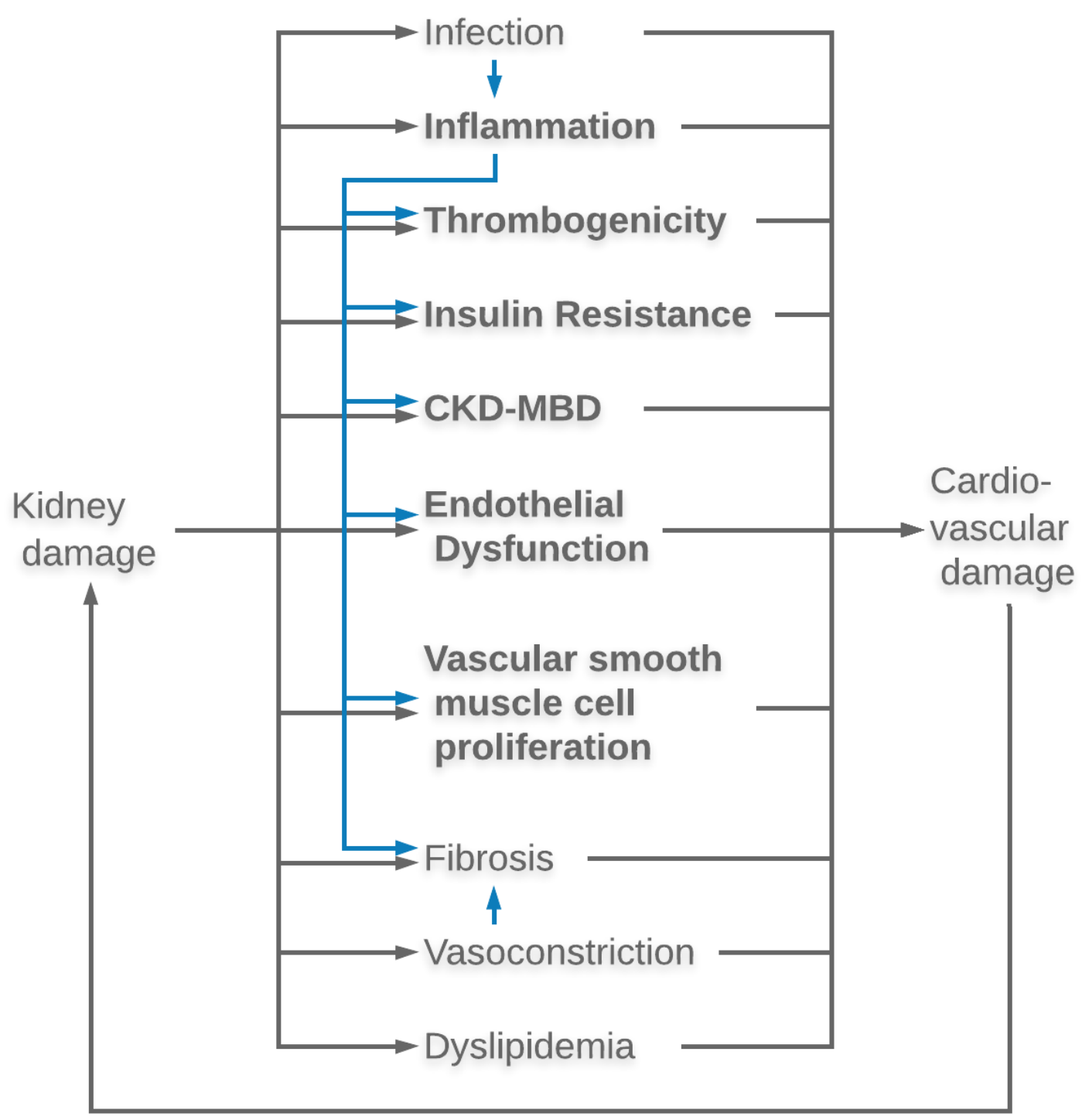


Figure 2

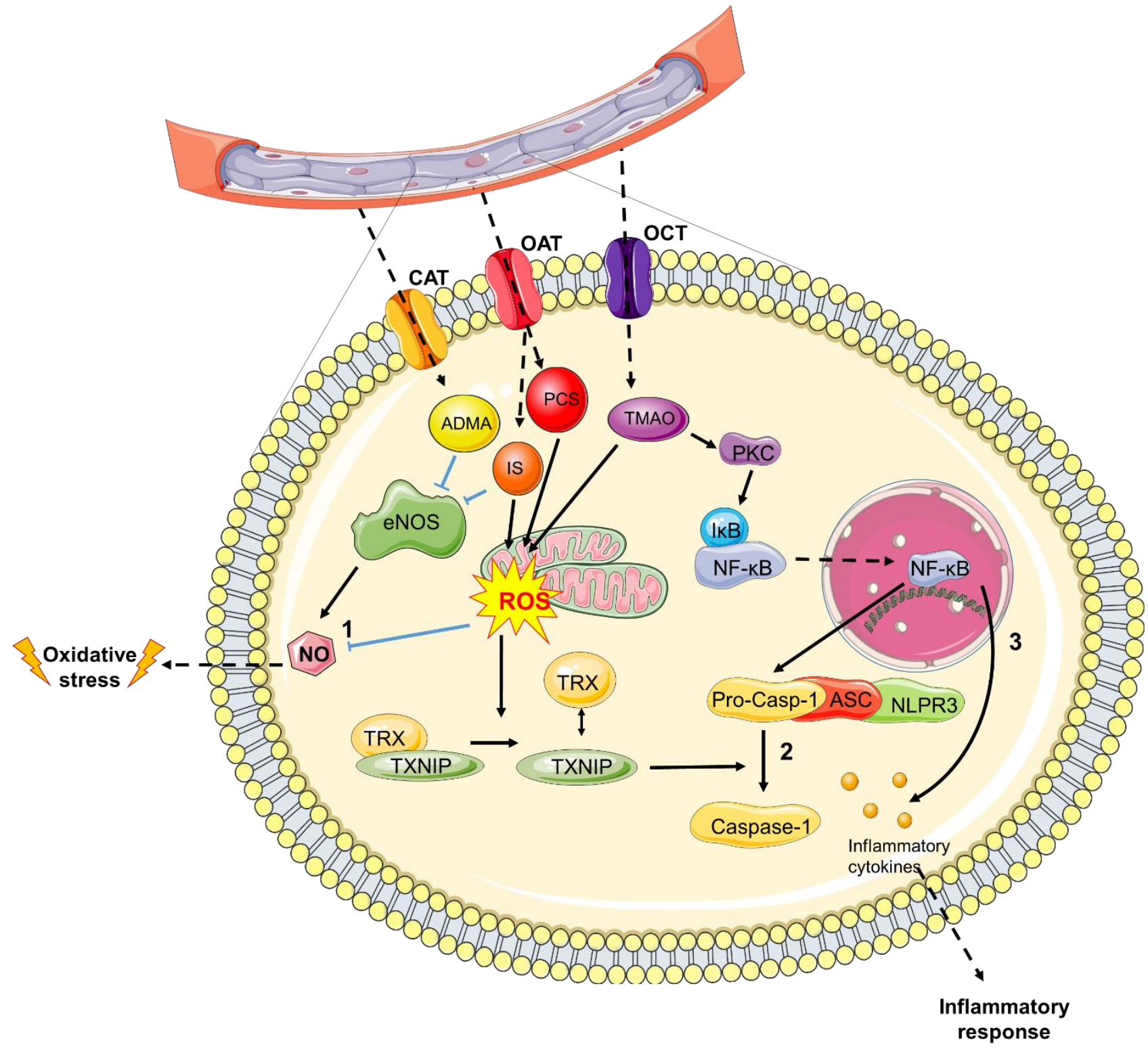


Table 1: Different stages of chronic kidney disease (CKD) and their respective symptoms, as classified by Kidney Disease: Improving Global Outcomes (KDIGO).

\begin{tabular}{|l|l|l|l|}
\hline Stage & $\begin{array}{l}\text { Kidney function } \\
\mathbf{m l} / \mathbf{m i n} / \mathbf{1 . 7 3} \mathbf{~ m}^{\mathbf{2}}\end{array}$ & Kidney damage & Symptoms \\
\hline No CKD & $\geq 90$ & None & No symptoms \\
\hline CKD 1 & $\geq 90$ & Slight & No symptoms \\
\hline CKD 2 & $89-60$ & Worrying & No symptoms \\
\hline CKD 3 & $59-30$ & Serious & $\begin{array}{l}\text { No symptoms, or sometimes } \\
\text { Edema } \\
\text { Polyuria } \\
\text { Hypertension } \\
\text { Anemia } \\
\text { Bone diseases }\end{array}$ \\
\hline CKD 4 & $29-15$ & Severe & $\begin{array}{l}\text { Same as CKD 3a \& CKD3b, } \\
\text { symptoms are more severe }\end{array}$ \\
\hline CKD 5 & $<15$ & Life threatening & $\begin{array}{l}\text { Same as CKD 4 plus } \\
\text { Itching } \\
\text { Muscle cramps } \\
\text { Anorexia } \\
\text { Nausea } \\
\text { Edema } \\
\text { Dyspnea } \\
\text { Sleep disturbances }\end{array}$ \\
\hline & & & \\
\hline
\end{tabular}

Each of the categories of eGFR (G1-G5) can also be categorized based on albumin/creatinine ratio into catergories of A1 (<30mg/g), A2 (30$300)$, and (>300). Abbreviation: CKD: chronic kidney disease; eGFR: estimated glomerular filtration rate. 
Table 2: Involvement of the discussed uremic toxins and their respective cardiovascular effects

\begin{tabular}{|c|c|c|c|c|c|c|}
\hline & $\begin{array}{l}\text { Endothelial } \\
\text { dysfunction }\end{array}$ & $\begin{array}{l}\text { Smooth } \\
\text { muscle } \\
\text { cells }\end{array}$ & $\begin{array}{l}\text { Leukocyte } \\
\text { activation }\end{array}$ & $\begin{array}{l}\text { CKD- } \\
\text { MBD }\end{array}$ & $\begin{array}{l}\text { Insulin } \\
\text { resistance }\end{array}$ & $\begin{array}{l}\text { Thrombo- } \\
\text { genicity }\end{array}$ \\
\hline \multicolumn{7}{|l|}{$\begin{array}{l}\text { Small water } \\
\text { soluble } \\
\text { compounds }\end{array}$} \\
\hline TMAO & $v$ & & $\boldsymbol{V}$ & & & \\
\hline ADMA & $v$ & $v$ & & & $v$ & \\
\hline SDMA & & & $v$ & & & \\
\hline Urea & $v$ & & & & $v$ & \\
\hline Uric acid & & & & & 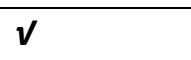 & $\sqrt{ }$ \\
\hline \multicolumn{7}{|l|}{$\begin{array}{l}\text { Middle } \\
\text { molecules }\end{array}$} \\
\hline FGF-23 & & & & $v$ & & \\
\hline \multicolumn{7}{|l|}{ Cytokines } \\
\hline IL-1 $\beta$ & $v$ & $v$ & & & & \\
\hline IL-6 & $v$ & $v$ & $\boldsymbol{V}$ & & $v$ & \\
\hline TNF- $\alpha$ & $v$ & $v$ & $v$ & & & \\
\hline \multicolumn{7}{|l|}{$\begin{array}{l}\text { Protein } \\
\text { bound } \\
\text { compounds }\end{array}$} \\
\hline $\begin{array}{l}\text { Indoxyl } \\
\text { sulfate }\end{array}$ & $v$ & $v$ & $v$ & $v$ & & $v$ \\
\hline $\begin{array}{l}\text { Indole 3- } \\
\text { acetic acid }\end{array}$ & $v$ & & $v$ & & $v$ & $v$ \\
\hline Kynurenine & & & & & & $\sqrt{v}$ \\
\hline $\begin{array}{l}\mathrm{p} \text {-Cresyl } \\
\text { sulfate }\end{array}$ & $v$ & $v$ & & $v$ & $v$ & \\
\hline $\begin{array}{l}\mathrm{p} \text {-Cresyl } \\
\text { glucuronide }\end{array}$ & & & $v$ & & & \\
\hline
\end{tabular}

Abbreviations: CKD-MBD, chronic kidney disease- mineral bone disease; TMAO, trimethylamine-N-oxide; ADMA, asymmetric dimethylarginine; SDMA, symmetric dimethylarginine; FGF-23, fibroblast growth factor-23; IL, interleukin; TNF- $\alpha$, tumor necrosis factor-alpha 
Table 3: solutes with the highest toxicity ranking

\begin{tabular}{|c|c|c|c|}
\hline $\begin{array}{l}\text { Highest clinical } \\
\text { evidence level }\end{array}$ & $\begin{array}{c}\text { Experimental } \\
\text { evidence score }\end{array}$ & $\begin{array}{c}\text { Second highest } \\
\text { clinical evidence level }\end{array}$ & $\begin{array}{c}\text { Experimental } \\
\text { evidence score }\end{array}$ \\
\hline p-Cresyl sulfate & 7 & $\begin{array}{l}\text { Advanced glycation } \\
\text { end products }\end{array}$ & 7 \\
\hline$\beta_{2}$-microglobulin & 6 & Indoxyl sulfate & 6 \\
\hline $\begin{array}{l}\text { Asymmetric dimethyl } \\
\text { arginine }\end{array}$ & 5 & Uric acid & 6 \\
\hline Kynurenines & 5 & Ghrelin & 5 \\
\hline $\begin{array}{l}\text { Carbamylated } \\
\text { compounds }\end{array}$ & 3 & Indole acetic acid & 5 \\
\hline $\begin{array}{l}\text { Fibroblast growth } \\
\text { factor-23 }\end{array}$ & 3 & Parathyroid hormone & 5 \\
\hline Interleukin-6 & 3 & Phenyl acetic acid & 5 \\
\hline $\begin{array}{l}\text { Tumor necrosis } \\
\text { factor- } \alpha\end{array}$ & 3 & $\begin{array}{l}\text { Trimethyl amine-N- } \\
\text { oxide }\end{array}$ & 5 \\
\hline \multirow[t]{7}{*}{$\begin{array}{l}\text { Symmetric dimethyl } \\
\text { arginine }\end{array}$} & 2 & $\begin{array}{l}\text { Rerinol binding } \\
\text { protein }\end{array}$ & 4 \\
\hline & & Endothelin & 3 \\
\hline & & $\begin{array}{l}\text { Immunoglobulin light } \\
\text { chains }\end{array}$ & 3 \\
\hline & & Interleukin-1 $\beta$ & 3 \\
\hline & & Interleukin-8 & 3 \\
\hline & & Neuropeptide $Y$ & 3 \\
\hline & & Lipids \& lipoproteins & 2 \\
\hline
\end{tabular}

Table modified from? ${ }^{7}$. Uremic toxins are divided into 2 groups based on the degree of clinical evidence and are within the groups ranked based on the degree of experimental evidence (high to low) Normal font, small water-soluble compounds; italics, protein-bound compounds; bold, larger middle molecules. Clinical: studies in patients relating toxin concentration to clinical outcomes. Experimental: in vitro or animal studies assessing the impact of toxins and their concentrations on biological effectsthat are part of the uremic syndrome. Abbreviations: FGF-23, fibroblast growth factor-23; IL, Interleukin; TNF-a, tumor necrosis factor-alpha.Modified from Vanholder R, Pletinck A, Schepers E, Glorieux G. Biochemical and Clinical Impact of Organic UremicRetention Solutes: A Comprehensive Update. Toxins. 2018;10:33. 